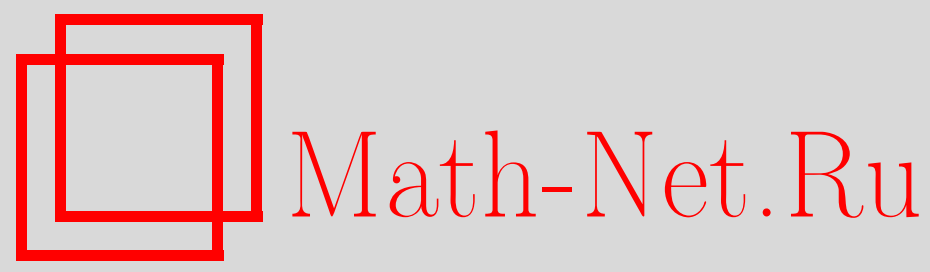

Н. А. Шананин, О распространении инвариантности ростков решений слабо нелинейных дифференциальных уравнений со взвешенными производными, Матем. заметки, 2002, том 71, выпуск 1, 135-143

DOI: https://doi.org/10.4213/mzm335

Использование Общероссийского математического портала Math-Net.Ru подразумевает, что вы прочитали и согласны с пользовательским соглашением http://www.mathnet.ru/rus/agreement

Параметры загрузки:

IP : 54.224 .135 .184

26 апреля 2023 г., 12:04:28

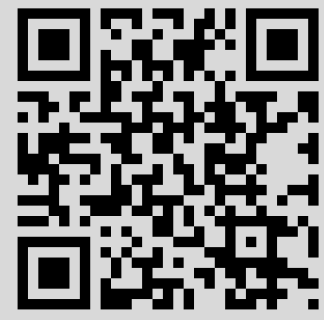




\title{
О РАСПРОСТРАНЕНИИ ИНВАРИАНТНОСТИ РОСТКОВ РЕШЕНИЙ СЛАБО НЕЛИНЕЙНЫХ ДИФФЕРЕНЦИАЛЬНЫХ УРАВНЕНИЙ СО ВЗВЕШЕННЫМИ ПРОИЗВОДНЫМИ
}

\section{Н. А. Шананин}

\begin{abstract}
Статья содержит теоремы о распространении инвариантности ростков решений для одного класса слабо нелинейных дифференциальных уравнений. В рассматриваемый класс уравнений входят модельные уравнения распространения нелинейных волн: нелинейное уравнение Шрёдингера, уравнение Кортевега-де Фриза и другие.

Библиография: 8 названий.
\end{abstract}

Из теоремы И.Г. Петровского [1] об аналитичности достаточно гладких решений аналитических эллиптических систем дифференциальных уравнений вытекает, что локальная инвариантность относительно групшы симметрий системы ростка решения в какой-либо точке области влечет инвариантность ростков данного решения во всех точках этой области. Обобщению этого свойства на один класс слабо нелинейных, неэллиптических уравнений с неаналитическими коэффициентами посвящена настоящая статья. В рассматриваемьй класс уравнений входят модельные уравнения распространения нелинейных волн: нелинейное уравнение Шрёдингера, уравнение Кортевега-де другие. Решения уравнений этого класса, вообще говоря, не обладают свойством аналитичности (в том числе по части переменных). Однако множество решений каждого из уравнений образует квазианалитический класс функций по части переменных. Это свойство составляет основу доказательства полученных ниже утверждений о распространении инвариантности ростков решений.

1. Параметрические семейства решений. Пусть в открытом множестве $\Omega \subset \mathbb{R}^{n}$ определен линейный дифференциальньй оператор со взвешенными производными

$$
P(x, D)=\sum_{\langle\varrho, \alpha\rangle \leqslant m} a_{\alpha}(x) D^{\alpha}, \quad D_{j}=\frac{1}{i} \frac{\partial}{\partial x_{j}}, \quad j=1,2, \ldots, n,
$$

где $\alpha=\left(\alpha_{1}, \alpha_{2}, \ldots, \alpha_{n}\right)$ - целочисленный неотрицательньй мультииндекс, веса $\varrho=\left(\varrho_{1}\right.$, $\left.\varrho_{2}, \ldots, \varrho_{n}\right)$ - натуральные числа, $\langle\varrho, \alpha\rangle-$ взвешенный порядок производной $D^{\alpha}:\langle\varrho, \alpha\rangle=$ $\varrho_{1} \alpha_{1}+\varrho_{2} \alpha_{2}+\cdots+\varrho_{n} \alpha_{n}$. Обозначим через $\mu$ минимальный вес: $\mu=\min \varrho_{j}$. Будем предполагать, что коэффициенты $a_{\alpha}(x) \in L_{\infty, \text { loc }}(\Omega)$ при $\langle\varrho, \alpha\rangle \leqslant m-\mu$ и $a_{\alpha}(x) \in C^{\infty}(\Omega)$

Работа выполнена при поддержке Российского фонда фундаментальных исследований, грант № 99-01-01139. 
при $m-\mu<\langle\varrho, \alpha\rangle \leqslant m$, причем по крайней мере один из коэффициентов $a_{\alpha}(x)$ при $\langle\varrho, \alpha\rangle=m$ отличен от тождественного нуля. В этом случае число $m$ называется взвеиенным порядком оператора.

Полный символ дифференциального оператора (1) является суммой квазиоднородных по $\xi \in \mathbb{R}^{n}$ слагаемых $p_{k}(x, \xi)=\sum_{\langle\varrho, \alpha\rangle=k} a_{\alpha}(x) \xi^{\alpha}$, степеней квазиоднородности $k=0,1, \ldots, m$, соответственно, $(x, \xi) \in \Omega \times \mathbb{R}^{n}$. Символ максимальной степени $p_{m}(x, \xi)$ назьвается взвешенным главным символом оператора $P(x, D)$.

Координаты $x=\left(x_{1}, \ldots, x_{n}\right)$ разделим на две части $x^{\prime}=\left\{x_{j} \mid \varrho_{j}=\mu\right\}$ и $x^{\prime \prime}=\left\{x_{j} \mid\right.$ $\left.\varrho_{j}>\mu\right\}$ соответственно, $\xi=\left(\xi^{\prime}, \xi^{\prime \prime}\right)$. Пусть $n^{\prime}\left(n^{\prime \prime}\right)$ - количество координатных функций в совокупности $x^{\prime}$ (соответственно $x^{\prime \prime}$ ). Оператор $P$ назьвается $\xi^{\prime}$-квазиэллиптическим в точке $x \in \Omega$, если из равенств $p_{m}(x, \xi)=0$ и $\xi^{\prime \prime}=0$ следует $\xi^{\prime}=0$. Оператор $P$ назьвается $\xi^{\prime}$-квазиэллиптическим в $\Omega$, если он является $\xi^{\prime}$-квазиэллиптическим во всех точках $\Omega$. Гиперповерхность $S=\{x \in U \mid \varphi(x)=0\}$, где $U$ - область в $\Omega, \varphi(x)-$ вешественная $C^{\infty}$-функция и $d \varphi \neq 0$ в $U$, назьвается нехарактеристической в точке $x^{0} \in S$ для оператора $P(x, D)$, если $p_{m}\left(x^{0}, \varphi_{x^{\prime}}\left(x^{0}\right), 0\right) \neq 0$. Очевидно, если оператор $P$ является $\xi^{\prime}$-квазиэллиптическим в $x^{0}$, то для того чтобы $S$ была нехарактеристической в $x^{0}$, необходимо и достаточно, чтобы $\varphi_{x^{\prime}}\left(x^{0}\right) \neq 0$.

Пусть оператор $P$ является $\xi^{\prime}$-квазиэллиптическим в $\Omega$. Тогда для любых $x^{0} \in \Omega$, $\eta^{\prime 0} \neq 0$ и $\xi^{0}=\left(\xi^{\prime 0}, \xi^{\prime \prime 0}\right)$ многочлен $p_{m}\left(x^{0}, \xi^{\prime 0}+z \eta^{\prime 0}, \xi^{\prime \prime 0}\right)$ относительно $z \in \mathbb{C}$ имеет степень $m / \mu$.

Предположим, что

1) оператор $P$ является $\xi^{\prime}$-квазиэллиптическим в $\Omega$ и для любых точки $x^{0} \in \Omega$ и неколлинеарных векторов $\left(\eta^{\prime 0}, 0\right)$ и $\left(\xi^{\prime 0}, \xi^{\prime \prime 0}\right)$ многочлен $p_{m}\left(x^{0}, \xi^{\prime 0}+z \eta^{\prime 0}, \xi^{\prime \prime 0}\right)$ имеет $m / \mu$ простых корней.

С оператором $P(x, D)$ свяжем пучок стариих символов

$$
\mathscr{H}_{P}(x, \xi, h)=\sum_{k=0}^{\mu-1} h^{k} p_{m-k}(x, \xi), \quad h \in \mathbb{R} .
$$

Пучок символов $\mathscr{H}_{P}(x, \xi, h)$ является многочленом по совокупности переменных $(\xi, h)$ с бесконечно дифференцируемьми по $x$ коэффициентами и $p_{m}(x, \xi)=\mathscr{H}_{P}(x, \xi, 0)$.

В силу теоремы о неявной функции для любой точки $\left(x^{0}, \eta^{\prime 0}, \xi^{0}, 0\right) \in \Omega \times \mathbb{R}_{\eta^{\prime}}^{n_{1}} \times$ $\mathbb{R}_{\xi}^{n} \times \mathbb{R}_{h}$, где $n_{1}$ - число переменных $\eta^{\prime}$ и векторы $\left(\eta^{\prime 0}, 0\right)$ и $\left(\xi^{\prime 0}, \xi^{\prime \prime 0}\right)$ не коллинеарны, найдется окрестность $W$, в которой уравнение $\mathscr{H}_{P}\left(x, \xi^{\prime}+z \eta^{\prime}, \xi^{\prime \prime}, h\right)=0$ относительно $z \in \mathbb{C}$ имеет $m / \mu$ простых корней $z_{j}\left(x, \eta^{\prime}, \xi, h\right)$.

Предположим, что

2) каждый корень $z_{j}\left(x, \eta^{\prime}, \xi, h\right)$ удовлетворяет одному из условий:

a) $\operatorname{Im} z_{j}\left(x^{0}, \eta^{\prime 0}, \xi^{0}, 0\right) \neq 0$;

b) $\operatorname{Im} z_{j}\left(x, \eta^{\prime}, \xi, h\right) \equiv 0$ в некоторой окрестности точки $\left(x^{0}, \eta^{\prime 0}, \xi^{0}, 0\right)$.

Пусть $G$ - ограниченное открытое множество в $\mathbb{R}^{n}$. Говорят, что комплекснозначная (вещественнозначная) функция $f(x, u)$, определенная для почти всех $x \in G$ и всех $u \in \mathbb{C}^{N}$ (соответственно $\mathbb{R}^{N}$ ), обладает свойством Каратеодори, если для почти всех $x \in G$ она непрерьвна по $u$ и для всех $u$ измерима по $x$. Ниже, чтобы не дублировать формулировки, будем рассматривать случай $u \in \mathbb{C}^{N}$. Если функция $f(x, u)$ обладает свойством Каратеодори, то для любых измеримых на $G$ функций $\left(u_{1}(x), u_{2}(x), \ldots\right.$, $\left.u_{N}(x)\right)=u(x)$ сложная функция $f(x, u(x))$ будет измеримой на $G[1$, теорема 18.3$]$. 
Для неограниченного открытого множества $\Omega$ будем говорить, что функция $f(x, u)$ обладает свойством Каратеодори, если она обладает этим свойством на любом множестве $G \times \mathbb{C}^{N}$, где $G$ - произвольное ограниченное открытое подмножество множества $\Omega$.

ОПРЕДЕЛЕниЕ 1 . Скажем, что функция $f(x, u)$ принадлежит классу $C L_{p}\left(\Omega \times \mathbb{C}^{N}\right)$ $\left(p \geqslant 1\right.$, кратко $\left.C L_{p}\right)$, если

1) $f(x, u)$ обладает свойством Каратеодори;

2) $f(x, 0) \in L_{p}(\Omega)$ и для любого компакта $K \subset \mathbb{C}^{N}$ найдется константа $C=C(K)$, с которой для всех $u^{1}$ и $u^{2} \in K$ и почти всех $x \in \Omega$ выполняется липшицева оценка

$$
\left|f\left(x, u^{1}\right)-f\left(x, u^{2}\right)\right| \leqslant C\left|u^{1}-u^{2}\right|
$$

Будем говорить, что функция $f(x, u)$ принадлежит классу $C L_{p, \text { loc, }}$ если для любой функции $\varphi(x) \in C_{0}^{\infty}(\Omega)$ произведение $\varphi(x) f(x, u)$ принадлежит классу $C L_{p}$. Если $f(x, u) \in C L_{p, \mathrm{loc}}$, то для любого фиксированного $u$ справедливо включение $f(x, u) \in$ $L_{p, \text { lос }}(\Omega)$. Более того, из $(2)$ следует, что для любых компактов $K_{1} \subset \Omega$ и $K_{2} \subset \mathbb{C}^{N}$ с некоторой константой $C=C\left(K_{1}, K_{2}\right)$ для почти всех $x \in K_{1}$ и всех $u \in K_{2}$ вьполняется оценка

$$
|f(x, u)| \leqslant|f(x, 0)|+C .
$$

Пусть $J$ - подмножество множества мультииндексов, удовлетворяющих неравенству $\langle\varrho, \alpha\rangle \leqslant m-\mu$, и $N(J)-$ их число. Если для каждого $\alpha \in J$ производная $\partial^{\alpha} u(x)$ обобщенной функции $u(x)$ принадлежит пространству $L_{\infty, \operatorname{loc}}(\Omega)$, то векторнозначная функция $\partial_{J} u(x)=\left(\partial^{\alpha} u(x)\right)_{\alpha \in J}$ принадлежит декартовому произведению $N(J)$ экземпляров пространства $L_{\infty, \text { loc }}(\Omega)$. Теперь если $f\left(x, u_{J}\right) \in C L_{p, \text { loc }}$, где $u_{J}=\left(u_{\alpha}\right)_{\alpha \in J}$, то из (3) следует, что сложная функция $f\left(x, \partial_{J} u(x)\right)$ принадлежит пространству $L_{p, \text { loc }}(\Omega)$. Если $u^{1}(x)$ и $u^{2}(x)$ - две функции, обладаюшие указанньми свойствами гладкости, то для любого компакта $K_{1} \subset \Omega$ найдется компакт $K_{2} \subset \mathbb{C}^{N(J)}$ такой, что для почти всех $x \in K_{1}$ справедливо включение $\partial_{J} u^{j}(x) \in K_{2}, j=1,2$. Из неравенства (2) вытекает, что найдется константа $C$, с которой для почти всех $x \in K_{1}$ выполняется оценка

$$
\left|f\left(x, \partial_{J} u^{1}(x)\right)-f\left(x, \partial_{J} u^{2}(x)\right)\right| \leqslant C \sum_{\alpha \in J}\left|\partial^{\alpha}\left(u^{1}(x)-u^{2}(x)\right)\right| \cdot
$$

Скажем, что обобщенная функция $u(x)$ принадлежит пространству $H_{w, \text { loc }}^{m-\mu}(\Omega)$, если для любого мультииндекса $\alpha \in\left(Z_{+}\right)^{n}$, взвешенньй порядок которого не превосходит $m-\mu$, производные $\partial^{\alpha} u(x)$ принадлежат пространству $L_{2, l o c}(\Omega)$. Будем говорить, что обобщенная функция $u(x)$ принадлежит пространству $L_{\infty, J, l o c}(\Omega)$, если $\partial^{\alpha} u(x) \in$ $L_{\infty, \text { loc }}(\Omega)$ при $\alpha \in J$.

Пусть $x^{0} \in \Omega$, иисло $d>0$ и $V=\left\{x^{\prime \prime}|| x^{\prime \prime}-x^{\prime \prime 0} \mid<d\right\} \times \Omega_{1} \subseteq \Omega$, где $\Omega_{1}-$ связное, открытое множество в $\mathbb{R}^{n^{\prime}}$, содержащее $x^{\prime 0}$. Из [5, теоремы 1] следует

ПРЕДЛОЖЕНИЕ 1. Предположим, что оператор $P(x, D)$ удовлетворяет в области $V$ условиям (1) $и(2)$ и $f\left(x, u_{J}\right) \in C L_{2, \mathrm{loc}}\left(V \times \mathbb{C}^{N(J)}\right)$. Тогда два решения $u^{1}(x) u u^{2}(x) \in H_{w, \mathrm{loc}}^{m-\mu}(V) \cap L_{\infty, J, \operatorname{loc}}(V)$ уравнения

$$
P(x, D) u=f\left(x, \partial_{J} u(x)\right)
$$


$x \in V$, совпадающие на мнохестве $\left\{x^{\prime \prime}|| x^{\prime \prime}-x^{\prime \prime 0} \mid<d\right\} \times U_{0} \subset V$, дде $U_{0} \subseteq \Omega_{1}-$ открытая окрестность $x^{\prime 0}$, совпадают в $V$.

Равенство (5) в предложении понимается в смысле теории обобщенных функций. Его левая часть есть результат действия линейного дифференциального оператора на обобщенную функцию. Правая часть представляет собой сложную функцию и при сделанных предположениях есть элемент пространства локально суммируемых в квадрате функций.

Будем говорить, что в открытом множестве $\mathscr{U} \in \mathbb{R}^{k} \times \Omega$ определено $k$-napaмeтрuческое семейство обобщенных функиий $\{u(s, x)\}$, если для любого непустого открытогоподмножества вида $V \times U \subseteq \mathscr{U}$, где $V$ - открытое подмножествов $\mathbb{R}^{k}$ и $U$-открытое подмножество в $\Omega$, определено семейство обобщенных функций $u(s, x) \in \mathscr{D}^{\prime}(U)$, зависящих от $s \in V$ как от параметра, причем если $V_{1} \times U_{1} \cap V_{2} \times U_{2} \neq \varnothing$, то $u_{1}(s, x)=u_{2}(s, x)$ в $U_{1} \cap U_{2}$ для всех $s \in V_{1} \cap V_{2}$. Множества вида $V \times U$ будем назьвать картами.

ОПРЕДЕЛЕНИЕ 2. Будем говорить, что росток в точке $\left(s^{0}, x^{0}\right) \in \mathscr{U} k$-параметрического семейства обобщенных функций $\{u(s, x)\}$ локально инвариантен относительно изменений значений параметров, если существует такая карта $V \times U$, содержащая $\left(s^{0}, x^{0}\right)$, что $u(s, x)=u\left(s^{0}, x\right)$ в $U$ для всех $s \in V$.

Положим $\Omega(s)=\{x \in \Omega \mid(s, x) \in \mathscr{U}\}, s \in \mathbb{R}^{k}$. Если $\Omega(s) \neq \varnothing$, то $k$-параметрическое семейство $\{u(s, x)\}$ определяет в $\Omega(s)$ обобщенную функцию $u^{s}(x)=u(s, x)$.

ОПРЕДЕЛЕНИЕ 3. Будем говорить, что семейство $\{u(s, x)\}$ есть $k$-napaмeтрическое семейство решений уравнения (5), если для любого непустого $\Omega(s)$

$$
u^{s}(x) \in H_{w, \operatorname{loc}}^{m-\mu}(\Omega(s)) \cap L_{\infty, J, \operatorname{loc}}(\Omega(s))
$$

и является решением уравнения на множестве $\Omega(s)$.

Пусть $x^{0} \in \Omega\left(s^{0}\right)$. Обозначим через $\mathscr{F}_{x^{0}}\left(\Omega\left(s^{0}\right)\right)$ связную компоненту слоя $\left\{x \in \Omega\left(s^{0}\right) \mid\right.$ $\left.x^{\prime \prime}=x^{\prime \prime 0}\right\}$, содержащую $x^{0}$.

Теорема 1. Предполохиим, что оператор $P(x, D)$ удовлетворяет условиям (1) $u(2)$ u $f\left(x, u_{J}\right) \in C L_{2, \text { loc }}\left(\Omega \times \mathbb{C}^{N(J)}\right)$. Тогда если росток $k$-параметрического семейства решений уравнения (5) в точке $\left(s^{0}, x^{0}\right) \in \mathscr{U}$ является локально инвариантным относительно изменений значений параметров, то и во всех точках множества $\mathscr{F}_{x^{0}}\left(\Omega\left(s^{0}\right)\right)$ ростки семейства решений $\{u(s, x)\}$ являются локально инвариантными относительно изменений значений параметров.

ДокАЗАТЕЛЬСтво. Для каждой точки $\left(s^{0}, x^{\prime \prime 0}, x^{\prime}\right) \in \mathscr{F}_{x^{0}}\left(\Omega\left(s^{0}\right)\right)$ найдутся открытые окрестности $V_{x^{\prime}} \subset \mathbb{R}^{k}$ точки $s^{0}, U_{x^{\prime}}^{\prime \prime} \subset \mathbb{R}^{n^{\prime \prime}}$ точки $x^{\prime \prime 0}$ и $U_{x^{\prime}}^{\prime} \subset \mathbb{R}^{n^{\prime}}$ точки $s^{0}$ такие, что $V_{x^{\prime}} \times U_{x^{\prime}}^{\prime \prime} \times U_{x^{\prime}}^{\prime} \subset \mathscr{U}$. Пусть $\left(s^{0}, x^{\prime \prime 0}, y^{\prime}\right) \in \mathscr{F}_{x^{0}}\left(\Omega\left(s^{0}\right)\right)$-произвольная точка множества $\mathscr{F}_{x^{0}}\left(\Omega\left(s^{0}\right)\right)$. Вследствие связности множества $\mathscr{F}_{x^{0}}\left(\Omega\left(s^{0}\right)\right)$ существует непрерьвньй путь $\gamma:[0,1] \rightarrow \mathscr{F}_{x^{0}}\left(\Omega\left(s^{0}\right)\right)$, соединяющий точки $\left(s^{0}, x^{\prime \prime 0}, x^{\prime 0}\right)$ и $\left(s^{0}, x^{\prime \prime 0}, y^{\prime}\right)$. Из покры-

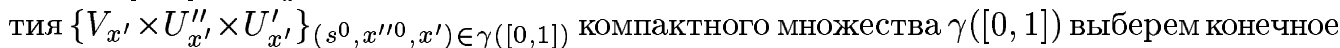
подпокрытие $\left\{V_{x^{\prime}(j)} \times U_{x^{\prime}(j)}^{\prime \prime} \times U_{x^{\prime}(j)}^{\prime}\right\}_{j=1}^{N}$. Пересечение $V=\bigcap_{j=1}^{N} V_{x^{\prime}(j)}$ есть открытая окрестность точки $s^{0}$, а пересечение $U^{\prime \prime}=\bigcap_{j=1}^{N} U_{x^{\prime}(j)}^{\prime \prime}$ есть открытая окрестность точки $x^{\prime \prime 0}$. Объединение $\Omega_{1}=\bigcup_{j=1}^{N} U_{x^{\prime}(j)}^{\prime}$-открытое связное подмножество в $\mathbb{R}^{n^{\prime}}$. 
Для каждого $s \in V$ на $U^{\prime \prime} \times \Omega_{1}$ определена обобшенная функция $u^{s}(x)=u(s, x)$, которая является решением уравнения (5) на этом множестве. Сужая при необходимости окрестности $V$ и $U^{\prime \prime}$, можно считать, что на произведении $U^{\prime \prime}$ на некоторую открытую окрестность $U_{0}^{\prime} \subset \Omega_{1}$ точки $x^{\prime 0}$ для всех $s \in V$ вьполняется равенство $u^{s}(x)=u^{s^{0}}(x)$. Отсюда и из предложения 1 следует доказьваемое утверждение.

Пусть $g: H_{w, \text { loc }}^{m-\mu}(\Omega) \cap L_{\infty, J, \text { loc }}(\Omega) \rightarrow H_{w, \operatorname{loc}}^{m-\mu}\left(\Omega_{g}\right) \cap L_{\infty, J, \text { loc }}\left(\Omega_{g}\right)$, где $\Omega_{g}$ - открытое множество в $\mathbb{R}^{n}$. Будем говорить, что росток функции $u(x) \in H_{w, \mathrm{loc}}^{m-\mu}(\Omega) \cap L_{\infty, J, \mathrm{loc}}(\Omega)$ в точке $x^{0} \in \Omega$ инвариантен относительно отображсения $g$, если $x^{0} \in \Omega_{g}$ и $g(u)(x)=$ $u(x)$ в некоторой окрестности $x^{0}$. Будем говорить, что отображсение $g$ сохраняет решения уравнения (5), если $\Omega \cap \Omega_{g} \neq \varnothing$ и образ $g(u)(x)$ любого решения $u(x) \in$ $H_{w, \text { loc }}^{m-\mu}(\Omega) \cap L_{\infty, J, \text { loc }}(\Omega)$ уравнения $(5)$ является решением этого уравнения на множестве $\Omega \cap \Omega_{g}$. Следующее утверждение доказьвается аналогично теореме 1.

ПРЕДЛОЖЕНИЕ 2. Предположим, что оператор $P(x, D)$ удовлетворяет в $\Omega$ условиям (1) $u(2)$ и $f\left(x, u_{J}\right) \in C L_{2, \operatorname{loc}}\left(V \times \mathbb{C}^{N(J)}\right)$. Тогда если в точке $x^{0}$ росток решения $u(x) \in H_{w, \operatorname{loc}}^{m-\mu}(\Omega) \cap L_{\infty, J, \operatorname{loc}}(\Omega)$ уравнения (5) инвариантен относительно отобрахения $g$, сохраняющего решения, то во всех точках мнохсества $\mathscr{F}_{x^{0}}\left(\Omega \cap \Omega_{g}\right)$ ростки $и(x)$ инвариантны относительно $g$.

В качестве примера рассмотрим в слое $\Omega=(0, T) \times \mathbb{R}^{n}$ слабо нелинейное уравнение теплопроводности

$$
u_{t}-\triangle u=f(u),
$$

где $f$ - непрерьвная функция комплексного аргумента, удовлетворяющая липшицевой оценке из определения 1. Введем взвешивание производных: однократному дифференцированию по $t$ присвоим вес 2 , а дифференцированию по $x-$ вес 1 . Линейная часть уравнения (6) удовлетворяет условиям (1) и (2).

Пусть $\tau \in(-T, T)$ и $a \in \mathbb{R}^{n}$. Положим $\Omega_{g}=(\tau, \tau+T) \times \mathbb{R}^{n}$ и $g(u)(t, x)=u(t+\tau, x+a)$. Очевидно, что отображение $g: H_{w, \text { loc }}^{1}(\Omega) \cap L_{\infty, \mathrm{loc}}(\Omega) \rightarrow H_{w, \mathrm{loc}}^{1}\left(\Omega_{g}\right) \cap L_{\infty, \mathrm{loc}}\left(\Omega_{g}\right)$ coxpaняет решения уравнения (6). Пусть $u(t, x) \in H_{w, \text { loc }}^{1}(\Omega) \cap L_{\infty, \mathrm{loc}}(\Omega)$ - решение уравнения (6), для которого в некоторой окрестности точки $\left(t^{0}, x^{0}\right) \in \Omega$ выполняется равенство $u(t+\tau, x+a)=u(t, x)$. Тогда в силу предложения 2 существует такое число $\varepsilon>0$, что указанное равенство выполняется в слое $\left(t^{0}-\varepsilon, t^{0}+\varepsilon\right) \times \mathbb{R}^{n}$. В частности, при $\tau=0$ отсюда следует, что в слое $\left(t^{0}-\varepsilon, t^{0}+\varepsilon\right) \times \mathbb{R}^{n}$ решение $u(t, x)$ либо постоянно, либо является периодическим по $x$.

2. Распространение инвариантности ростков $C^{\infty}$-решений относительно непрерывной локальной группы симметрий. Для уравнений вида (5), правая часть которых задается вещественнозначной функцией $f\left(x, u_{J}\right) \in C^{\infty}\left(\Omega \times \mathbb{R}^{N(J)}\right)$ и решение ищется в классе вещественнозначных бесконечно дифференцируемых функций, отправляясь от какого-либо частного $C^{\infty}$-решения, параметрические семейства $C^{\infty}$ решений можно строить с помощью теории симметрий. Параметрическое семейство решений определяется локальным действием некоторой группы Ли $G$ на решениях дифференциального уравнения [7], [8]. Решение $u(x)$ назьвается локально инвариантным относительно группы Ли $G$, если для любой точки $x \in \Omega$ существуют открытая окрестность $U_{x} \subseteq \Omega$ точки $x$ и открытая окрестность $G_{x}$ единицы групшы такие, что $g \circ u(x)=u(x)$ в $U_{x}$ для всех $g \in G_{x}$. Локализуем это понятие. Росток решения $u(x)$ в точке $x^{0}$ будем назьвать инвариантным относительно локального действия группь 
$Л u G$, если существуют открытая окрестность $U_{x^{0}} \subseteq \Omega$ точки $x^{0}$ и открытая окрестность $G_{x^{0}}$ единицы группы такие, что $g \circ u(x)=u(x)$ в $U_{x^{0}}$ для всех $g \in G_{x^{0}}$.

Теорема 2. Предположим, что оператор $P(x, D)$ в левой части дифференииального уравнения

$$
\sum_{m-\mu<\langle\varrho, \alpha\rangle \leqslant m} a_{\alpha}(x) \partial_{x}^{\alpha} u=f\left(x, \partial_{J} u(x)\right)
$$

имеет вещественные коэффичиенты $a_{\alpha}(x) \in C^{\infty}(\Omega)$ и удовлетворяет услови-

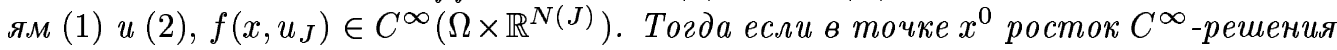
$u(x)$ является инвариантным относительно локального действия группь симметрий $G$ этого уравнения, то во всех точках мнохества $\mathscr{F}_{x^{0}}(\Omega)$ ростки решения $u(x)$ являются инвариантными относительно локального действия группь $G$.

ДокАЗАТЕЛЬСтво. Рассмотрим уравнение (5) с правой частью

$$
f_{1}\left(x, \partial_{J} u(x)\right)=f\left(x, \frac{1}{2}\left(\partial_{J} u(x)+\partial_{J} \bar{u}(x)\right)\right)
$$

где черта над функцией означает комплексное сопряжение. Поскольку

$$
f_{1}\left(x, u_{J}\right) \in C L_{2, \mathrm{loc}}\left(\Omega \times \mathbb{C}^{N(J)}\right)
$$

и $g \circ u(x)$ есть параметрическое семейство $C^{\infty}$-решений, то доказываемое утверждение есть следствие теоремы 1.

3. Распространение инвариантности ростков обобщенных решений. Для дифференциальных уравнений вида (5), инвариантных относительно сдвигов

$$
\sum_{\langle\varrho, \alpha\rangle \leqslant m} a_{\alpha} D^{\alpha} u(x)=f\left(\partial_{J} u(x)\right), \quad x \in \Omega,
$$

имеется естественњый способ построения параметрических семейств обобщенных решений. Пусть $\nu$ - ненулевой вектор в $\mathbb{R}^{n}$. Положим $\mathscr{U}=\{(s, x) \in \mathbb{R} \times \Omega \mid x, x+s \nu \in \Omega\}$. Тогда если $u(x) \in H_{w, \operatorname{loc}}^{m-\mu}(\Omega) \cap L_{\infty, J, \operatorname{loc}}(\Omega)$ есть обобщенное решение уравнения $(8)$, то $v(s, x)=u(x+s \nu)$ - однопараметрическое семейство обобщенных решений этого уравнения, определенное на множестве $\mathscr{U}$. Будем говорить, что росток решения $u(x)$ в точке $x^{0}$ инвариантен относительно локального действия группы сдвигов по направлению вектора $\nu$, если существуют открытая окрестность $U_{x^{0}} \subseteq \Omega$ точки $x^{0}$ и положительное число $\delta$ такие, что $u(x+s \nu)=u(x)$ в $U_{x^{0}}$ при $|s|<\delta$. Вследствие теоремы 1 имеем

ПРЕДЛОЖЕНИЕ 3. Предположим, что дифференциальный оператор $P(D)$ в левой части уравнения (8) удовлетворяет условиям (1) и (2) и непрерывная функиия $f\left(u_{J}\right)$, определенная на $\mathbb{C}^{N(J)}$, удовлетворяет липиицевой оценке из определения 1. Тогда если в точке $x^{0}$ росток решения $и(x)$ уравнения (8) инвариантен относительно локального действия группы сдвигов по направлению вектора $\nu$, 
то и во всех точках мнохсества $\mathscr{F}_{x^{0}}(\Omega)$ ростки решения $u(x)$ являются инвариантными относительно локального действия группы сдвигов по направлению вектора $\nu$.

Рассмотрим несколько примеров применения предложения 3. В уравнении типа Колмогорова-Петровского-Пискунова

$$
u_{t}-u_{x x}=f\left(u, u_{x}\right), \quad(t, x) \in \Omega \subseteq \mathbb{R}^{2},
$$

введем взвешивание производных: однократному дифференцированию по $t$ присвоим вес 2 , а дифференцированию по $x$ - вес 1 . Правая часть уравнения и решение предполагаются вещественнозначньми. Строго говоря, предложение 3 не применимо в рассматриваемом случае. Функция $f$ в правой части уравнения (8) должна быть определена для комплексных значений аргументов. Для того чтобы устранить это рассогласование, достаточно заменить правую часть в уравнении $(9)$ на $f\left((u+\bar{u}) / 2,\left(u_{x}+\bar{u}_{x}\right) / 2\right)$, где черта над функцией означает комплексное сопряжение. Нетрудно проверить, что линейная часть уравнения (9) удовлетворяет условиям (1) и (2). Из предложения 3 получаем

СлЕДСТВИЕ 1. Пусть непрерывная вещественнозначная функиия $f\left(u, u_{1}\right)$ вещественных переменных $и$ и $u_{1} \in \mathbb{R}$ удовлетворяет липиицевой оценке из определения 1 и вещественнозначная функиия $u(t, x)$, принадлежсащая классу

$$
\left\{u(t, x) \in L_{\infty, \mathrm{loc}}(\Omega) \mid u_{x}(t, x) \in L_{\infty, \mathrm{loc}}(\Omega)\right\}
$$

является решением уравнения (9). Тогда если росток решения $u(t, x)$ в точке $\left(t^{0}, x^{0}\right) \in \Omega$ инвариантен относительно локальных сдвигов по направлению вектора $\nu \in \mathbb{R}^{2} \backslash\{0\}$, то ростки этого решения инвариантны относительно таких сдвигов во всех точках связной компоненты мнохества $\left\{\left(t^{0}, x\right) \in \Omega\right\}$, содержащей точку $\left(t^{0}, x^{0}\right)$.

В частности, если $\Omega=\left(T_{1}, T_{2}\right) \times\left(a_{1}, a_{2}\right)$ и росток решения $u(t, x)$ в точке $\left(t^{0}, x^{0}\right) \in \Omega$ инвариантен относительно сдвигов по направлению $\nu=\left(\nu_{1}, \nu_{2}\right)$, то в силу следствия найдется положительное число $\varepsilon$ такое, что в слое $\left(t^{0}-\varepsilon, t^{0}+\varepsilon\right) \times\left(a_{1}, a_{2}\right)$ решение $u(t, x)$ имеет вид $u_{0}\left(\nu_{2} t-\nu_{1} x\right)$, где $u_{0}$ - обобщенная функция одного переменного. Нетрудно определить класс гладкости $u_{0}$.

ЗАмЕчАниЕ. Для решений уравнения типа нелинейного уравнения Шрёдингера

$$
-i u_{t}-u_{x x}=f\left(u, u_{x}\right)
$$

имеет место полный аналог следствия 1.

В уравнении типа уравнения Кортевега-де Фриза

$$
u_{t}+u_{x x x}=f\left(u, u_{x}\right)
$$

введем взвешивание производных: однократному дифференцированию по $t$ присвоим вес 3 , а дифференцированию по $x$ - вес 1 . Правая часть уравнения и решение так же, как и в предыдущем случае, предполагаются вещественнозначными. Оператор линейной части уравнения (11) удовлетворяет условиям (1) и (2). Из предложения 3 получаем 
СлЕДСТВИЕ 2. Пусть непрерывная вещественнозначная функция $f\left(u, u_{1}\right)$ вещественных переменных $и$ и $u_{1} \in \mathbb{R}$ удовлетворяет липиицевой оценке из определения 1 и вещественнозначная функиия $u(t, x)$, принадлежсащая классу

$$
\left\{u(t, x) \in L_{\infty, \mathrm{loc}}(\Omega) \mid u_{x}(t, x) \in L_{\infty, \mathrm{loc}}(\Omega), u_{x x}(t, x) \in L_{2, \mathrm{loc}}(\Omega)\right\}
$$

является решением уравнения (11). Тогда если росток решения $u(t, x)$ в точ$\kappa е\left(t^{0}, x^{0}\right) \in \Omega$ инвариантен относительно локальных сдвигов по направлению вектора $\nu \in \mathbb{R}^{2} \backslash\{0\}$, то ростки этого решения инвариантны относительно таких сдвигов во всех точках связной компоненты множества $\left\{\left(t^{0}, x\right) \in \Omega\right\}$, содержащей точку $\left(t^{0}, x^{0}\right)$.

Для уравнений типа уравнения Кадомцева-Петвиашвили

$$
u_{x_{1} x_{1} x_{1} x_{1}}+3 u_{x_{2} x_{2}}+u_{x_{1} x_{3}}=f\left(u, u_{x_{1}}, u_{x_{2}}, u_{x_{1} x_{1}}\right), \quad f \in C L_{2, \mathrm{loc}}
$$

и типа уравнения Буссинеска

$$
u_{t t}+u_{x x x x}=f\left(u, u_{x}, u_{x x}\right), \quad f \in C L_{2, \mathrm{loc}}
$$

справедливы аналоги следствий 1 и 2. В случае уравнения (12) достаточньй класс гладкости решений определяется включениями: $u_{x_{1}}, u_{x_{2}}, u_{x_{1} x_{1}} \in L_{\infty, \operatorname{loc}}(\Omega)$ и $u_{x_{3}}, u_{x_{1} x_{2}}$, $u_{x_{1} x_{1} x_{1}} \in L_{2, \text { lос }}(\Omega)$, а для уравнения (13) - включениями: $u_{x}, u_{x x} \in L_{\infty, \text { lос }}(\Omega), u_{t}$, $u_{x x x} \in L_{2, \mathrm{loc}}(\Omega)$.

Рассмотрим теперь следуюший вид действия групшы на обобщенные решения слабо нелинейных уравнений. Предположим, что в пространстве $\mathbb{R}^{n}$ определено действие группы $G$, причем для каждого фиксированного $g \in G$ соответствующее отображение $\varphi_{g}: \mathbb{R}^{n} \rightarrow \mathbb{R}^{n}$ есть $C^{\infty}$-диффеоморфизм. Образ открытого множества $\Omega \subseteq \mathbb{R}^{n}$ при отображении $\varphi_{g}$ обозначим через $\Omega_{g}$. Очевидно, $\left(\Omega_{g_{1}}\right)_{g_{2}}=\Omega_{g_{2} \cdot g_{1}}$. Предположим, что для любых $g \in G$ и открытого множества $\Omega$ определено непрерывное отображение $\Phi_{g, \Omega}: H_{w, \text { loc }}^{m-\mu}(\Omega) \cap L_{\infty, J, \text { loc }}(\Omega) \rightarrow H_{w, \text { loc }}^{m-\mu}\left(\Omega_{g}\right) \cap L_{\infty, J, \text { loc }}\left(\Omega_{g}\right)$, согласованное с групшвой операцией: $\Phi_{g_{1}, \Omega_{g_{2}}} \circ \Phi_{g_{2}, \Omega}=\Phi_{g_{1} \cdot g_{2}, \Omega}$. Будем говорить, что действие групшы есть локальная группа симметрий уравнения (5) (определенного в $\Omega$ ), если образ $\Phi_{g, \Omega}(u)$ любого решения $u(x) \in H_{w, \text { loc }}^{m-\mu}(\Omega) \cap L_{\infty, J, \text { loc }}(\Omega)$ уравнения (5) является решением этого уравнения на множестве $\Omega \cap \Omega_{g}$, если последнее не пусто. Пусть $G$ - групп Ли. Будем говорить, что росток обобщенного решения $u(x)$ в точке $x^{0}$ инвариантен относительно локального действия группы симметрий $G$, если существуют открытая окрестность $U_{x^{0}} \subseteq \Omega$ точки $x^{0}$ и открытая окрестность $G_{x^{0}}$ единицы группы такие, что $\Phi_{g, \Omega}(u)(x)=u(x)$ в $U_{x^{0}}$ для всех $g \in G_{x^{0}}$.

ТЕОрема 3. Предположим, что оператор $P(x, D)$ удовлетворяет условиям (1) $u(2), f\left(x, u_{J}\right) \in C L_{2, \text { loc }}\left(\Omega \times \mathbb{C}^{N(J)}\right)$ u $G$ есть локальная группа симметрий уравнения (5). Тогда если в точке $x^{0}$ росток обобщенного решения $u(x)$ уравнения $(5)$ является инвариантным относительно локального действия группы Ли $G$, то ростки этого решения являются инвариантными относительно локального действия группь Ли $G$ во всех точках мнохества $\mathscr{F}_{x^{0}}(\Omega)$. 
ДокАЗАТЕльство. Это утверждение следует из теоремы 1. В самом деле, по определению групш Ли для подходящего натурального числа $k$ сушествует диффеоморфизм $\kappa$ открытой окрестности $U$ начала координат в пространстве $\mathbb{R}^{k}$ на открытую окрестность единицы групшы. Положим $\mathscr{U}=\left\{(s, x) \in U \times \Omega \mid \varphi_{\kappa(s)}(x) \in \Omega\right\}$. Если $u(x)$ является обобщенньм решением уравнения $(5)$, то $\left\{\Phi_{\kappa(s), \Omega}(u)(x)\right\}$ есть $k$-параметрическое семейство решений уравнения (5), определенноена $\mathscr{U}$. Применение теоремы 1 завершает доказательство.

В нелинейном уравнении Шрёдингера

$$
-i u_{t}-u_{x x}=f\left(|u|^{2}\right) u, \quad(t, x) \in \Omega=(0, T) \times \mathbb{R},
$$

введем взвешивание производных: однократному дифференцированию по $t$ присвоим вес 2 , а дифференцированию по $x-$ вес 1 . Предположим, что функция $f(v) \in C\left(\overline{\mathbb{R}}_{+}\right)$ вещественнозначна и удовлетворяет липшицевой оценке из определения 1 . Пусть $G=\mathbb{R}$ и $\varphi_{s}(t, x)=(t, x-2 s t)$. Тогда $\varphi_{s}(\Omega)=\Omega$ для всех $s \in \mathbb{R}$. Пусть

$$
\Phi_{s, \Omega}(u)(t, x)=e^{i\left(x s-t s^{2}\right)} u(t, x-2 s t)
$$

при $s \in \mathbb{R}$. Нетрудно проверить, что введенное действие группы $\mathbb{R}$ является группой симметрий уравнения (14). В силу теоремы 3 если в точке $\left(t^{0}, x^{0}\right) \in \Omega$ росток решения $u(t, x)$ инвариантен относительно локального действия группы $\mathbb{R}$, то найдется такое число $\varepsilon>0$, что в слое $\Pi_{\varepsilon, t_{0}}=\left(t^{0}-\varepsilon, t^{0}+\varepsilon\right) \times \mathbb{R}$ решение $u(t, x)$ инвариантно относительно локального действия этой группы. Отсюда следует, что в полосе $\Pi_{\varepsilon, t_{0}}$ решение имеет вид

$$
\frac{C}{\sqrt{t}} \exp \left(i \frac{x^{2}}{4 t}+i \int_{t_{0}}^{t} f\left(\frac{|C|^{2}}{\tau}\right) d \tau\right)
$$

где $C \in \mathbb{C}$ - произвольная константа.

\section{СПИСОК ЦИТИРОВАННОЙ ЛИТЕРАТУРЫ}

[1] Петровский И. Г. Об аналитичности решений систем уравнений с частными производными. Избранные труды. Системы уравнений с частными производными. Алгебраическая геометрия. М.: Наука, 1986.

[2] Вайнберг М. М. Вариационные методы исследования нелинейных операторов. М., 1956.

[3] Calderón A. P. Uniqueness in Cauchy problem for partial differential equations // Amer. J. Math. 1958. V. 80. P. 16-36.

[4] Шананин Н. А. Об однозначном продолжении решений дифференциальных уравнений со взвешенными производными // Матем. сб. 2000. Т. 191. № 3. С. 113-142.

[5] Шананин Н. А. О частичной квазианалитичности обобщенных решений слабо нелинейных дифференциальных уравнений со взвешенными производными // Матем. заметки. 2000. Т. 68. № 4. C. $608-619$.

[6] Хермандер Л. Анализ линейных дифференциальных операторов с частными производными. Т. 1-4. М.: Мир, 1987.

[7] Олвер П. Приложения групп Ли к дифференциальным уравнениям. М.: Мир, 1989.

[8] Виноградов А. М., Красильщик И. С. и др. Симметрии и законы сохранения уравнений математической физики. М.: Наука, 1997. 